Clinical Imaging

Case report

\title{
A novel preoperative fusion analysis using 3-dimensional MDCT combined with 3-dimensional MRI for patients with hilar cholangiocarcinoma
}

\author{
Yukio Oshiro, M.D. ${ }^{\mathrm{a}}$, Ryoko Sasaki, M.D., Ph.D., F.I.C.S. ${ }^{\mathrm{a}, *}$, \\ Katsuhiro Nasu, M.D., Ph.D. ${ }^{\mathrm{b}}$, Nobuhiro Ohkohchi, M.D., Ph.D. ${ }^{\mathrm{a}}$
}

${ }^{a}$ Department of Organ Transplantation, Gastroenterology and Hepato-biliary surgery, University of Tsukuba, Graduate School of Comprehensive Human Sciences, 1-1-1 Tennodai, Tsukuba 305-8575, Japan

${ }^{b}$ Department of radiology, University of Tsukuba, Graduate School of Comprehensive Human Sciences, 1-1-1 Tennodai, Tsukuba 305-8575, Japan

*Corresponding address, Reprint request:

Ryoko Sasaki, M.D., Ph.D.

Department of Organ Transplantation, Gastroenterology and Hepato-biliary surgery, University of Tsukuba, Graduate School of Comprehensive Human Sciences, 1-1-1

Tennodai, Tsukuba 305-8575, Japan

TEL: +81-29-853-3221

FAX: +81-29-853-3222

Email: rsasaki.dr@hotmail.co.jp

\section{An abbreviated title:}

3-dimensional fusion MDCT combined with MRCP 


\section{Abstract}

2 The purpose of the present study was to evaluate the anatomical relationship between

3 the tumor, portal veins, hepatic arteries, and hilar hepatic ducts at the hepatic hilum

4 using a novel preoperative fusion analysis for patients with hilar cholangiocarcinoma.

5 This involved combining 3-dimensional multidetector-row computed tomography

6 (3D-MDCT) with 3-D magnetic resonance imaging (MRI). This novel fusion imaging

7 technique can play an important clinical role for patients undergoing surgery for hilar

8 cholangiocarcinoma.

9

10 Key words:

11 hilar cholangiocarcinoma, 3-dimensional imaging, fusion imaging, MDCT, MRI 


\section{Introduction}

To determine the optimal surgical procedure for patients with hilar cholangiocarcinoma, it is essential to know the anatomical relationship between the tumor and the vessels around the hepatic hilum in individual patients. Several institutions have recently developed 3-dimensional multidetector-row computed tomography (3D-MDCT) cholangiography or drip infusion 3D-MDCT for use with patients with hilar cholangiocarcinoma [1-4]. However, it has been said that these radiological examinations have several problems with side effects such as cholangitis or anaphylactic shock due to infusion of the contrast material into the vein or bile duct. Therefore, we were looking forward to a new modality and method. To our knowledge there have been no published reports concerning the preoperative visualization of 3D-MDCT fusion images combined with 3D-magnetic resonance cholangiopancreatography (MRCP) images. The purpose of the present study was to preoperatively evaluate portal veins and hilar hepatic ducts at the hepatic hilum in patients with hilar cholangiocarcinoma.

\section{Case}

The patient was a 52-year-old man. He consulted the Tsukuba University Hospital complaining of jaundice. Laboratory blood samples showed elevated total bilirubin and carcinogenic antigen (CA) 19-9, and MDCT and MRI were subsequently performed (Fig. 1).

MDCT images were acquired using an IDT-16 imager (Philips, Eindhoven, The Netherlands). Scan settings included a pitch of 17 , a 0.75 -second scan time per rotation, a table speed of $12 \mathrm{~mm} /$ rotation, and a detector configuration of $0.75 \times 16 \mathrm{~mm}$. A power injector was used to administer $100 \mathrm{~mL}$ iopamidol (370 mgI/mL) at $4 \mathrm{~mL} / \mathrm{s}$ through a 20-G high pressure intravenous catheter. The MDCT protocol obtained 3 sets of hepatic images in succession, including images of arterial, and those of portal venous, and hepatic venous phases. The arterial phase images were obtained 5 seconds after peak aortic enhancement, portal venous phase images were taken 70 seconds after starting the injection, and the hepatic venous phase began 180 seconds after starting the injection. The MRI was performed on a 1.5 Tesla MRI imager (Achieva Nova Dual, Philips Medical Systems, Eindhoven, The Netherlands)

The preoperative fusion examination consisted of 4 steps. We first combined heavily T2-weighted MRCP images with axial thin slice 3D-T1-turbo field echo (TFE) images. Second, we combined the thin TFE images with thin MDCT axial images. Third, we superimposed MRCP data onto the axial CT images, focusing on the area around the 
1 hepatic hilum. Forth, we checked the following three registration landmarks: 1) the right

2 border along the umbilical potion, 2) the bifurcation of the right and left hepatic arteries,

3 and 3) the bifurcation of the right and left portal veins. Although we were able to

4 delineate the hilar cholangiocarcinoma on the reconstructed 3D image by tracing the

5

6 enhanced tumor on the 2D MDCT, we were not able to detect the tumor on the MRCP as expected. However, we confirmed that the resultant 3D fusion image was correct by checking the three registration landmarks (Fig. 2).

We used the Synapse Vincent medical imaging system (Fujifilm Medical, Tokyo), which was developed specifically for 3D visualization and virtual resection of the liver. This software offers standardized computation of liver anatomy functions and volumetric risk analysis based on 2D-CT imaging. The structures of the liver, portal vein, hepatic artery, hepatic vein, and tumor were extracted from MDCT scan data. One of the most important points of this novel fusion imaging is to examine MDCT during the patient's expiratory phase, which is the same condition of MRI. The user can visualize 3D images of the structures mentioned above as well as those of the hepatic arterial, portal, and hepatic venous phases. These images can also be rotated separately or simultaneously (Fig. 2).

We diagnosed the patient's disease as locally advanced hilar cholangiocarcinoma, T2, N1, M0, Stage IIB according to the UICC-TNM classification [5], and Type IIIb based on the Bithmuth- Corlette classification [6]. The patient and his family agreed to this procedure and provided informed consent. The patient tolerated MDCT and MRI without serious complications such as skin rash or other allergic adverse effects.

A chief surgeon (R.S.) performed the surgical resection. Based on preoperative imaging, we determined that the most appropriate surgical procedure was left hepatic lobectomy combined with total caudate lobectomy and extrahepatic bile duct resection (Fig. 3), No concomitant vascular resection was performed. Operative time was 581 minutes, and estimated blood loss was $1215 \mathrm{ml}$. The patient's postoperative course was stable and uneventful, and he was discharged on postoperative day 14. Postoperative clinical pathology revealed that there was no residual carcinoma (R0). The patient is now alive 1 year and 1 month after surgery with no evidence of recurrence.

\section{Discussion}

To determine the optimal surgical approach to hilar cholangiocarcinoma for individual patients, it is essential to perform a detailed evaluation of the anatomical structures at the hepatic hilum. By using newly developed software and a novel method, we were able to easily analyze the hepatic arteries, portal veins, hepatic veins, and hilar 
1 bile ducts without performing 3D-MDCT cholangiography or drip infusion 3D-MDCT.

2 A more recent advance is the development of image integration, which refers to the

3 process of registering previously acquired MRI and CT scans of the heart with the

4 mapping space during an ablation procedure [7]. Further, a urology study has reported

5 the usefulness of CT-MRI fusion imaging in radiotherapy planning for localized prostate

6 cancer [8].

$7 \quad$ Several limitations of this novel modality should be addressed. First, at present this

8 approach requires a considerable amount of time (about 5-6 hours) to create detailed

9 images. Second, as described in the Patients and Methods section, obtaining images

10 during the experiratory phase is one of the most important aspects of this method.

11 Obtaining satisfactory images will be easiest with patients who are able to cooperate in

12 this aspect. Third, this study involved only one patient, and our results will therefore

13 need to be confirmed with larger populations.

14 In conclusion, this novel fusion imaging technique should play an important role in

15 preoperative planning for patients undergoing surgery for hilar cholangiocarcinoma.

\section{Acknowledgements}

The authors thank Mr. K. Kotera and Ms. K. Suzuki of Fujifilm Medical Co., Ltd, for

19 their kind support. 


\section{References}

1. Sasaki R, Kondo T, Oda T, et al. Impact of three-dimensional analysis of multidetector row computed tomography cholangiography in operative planning for hilar cholangiocarcinoma. Am J Surg 2011;202:441-448.

2. Takahashi K, Sasaki R, Kondo T, et al. Preoperative 3D volumetric analysis for liver congestion applied in a patient with hilar cholangiocarcinoma. Langenbecks Arch Surg 2010;395:761-765.

3. Endo I, Shimada H, Sugioka M, et al. Role of three-dimensional imaging in operative planning for hilar cholangiocarcinoma. Surgery 2007;142:666-675.

4. Hansen C, Wieferich J, Ritter F, et al. Illustrative visualization of 3D planning models for augmented reality in liver surgery. Int J comput Assist Radiol Surg 2010;2:133-141.

5. Greene FL, Page DL, Fleming ID, et al. AJCC Cancer Staging Manual, 6th edn. New York: Springer-Verlag, 2002.

6. Bithmuth $\mathrm{H}$, CorletteMB. Intrahepatic cholangioenteric anastomosis in carcinoma of the hilus of the liver. Surg Gynecol Obstet 1975;140:170-177.

7. Govil A, Calkins H, Spragg DD, et al. Fusion of imaging technologies: how, when, and for whom? J Interv Card Electrophysiol 2011;32:195-203.

8. Tanaka H, Hayashi S, Ohtakara K, et al. Usefulness of CT-MRI fusion in radiotherapy planning for localized prostate cancer. J Radiat Res 2011;52:782-788. 
3 Fig. 1 Axial MDCT and MRCP. (A) Axial MDCT demonstrates bilateral intrahepatic bile duct dilatation. (B) MRCP demonstrates that the bilateral intrahepatic bile ducts were dilated, and that the confluence of the right and left hepatic ducts is tapered and obstructed at the hepatic hilum.

Fig. 2 Preoperative fusion analysis using 3D-MDCT combined with 3D MRI imaging (A) and a virtual dissection line when performing left hepatic lobectomy (B). The red line indicates the planned dissection line. Novel modality was successfully described and the surgeon and surgical staff could determine the appropriate dissection line preoperatively.

Fig. 3 The actual liver cut surface shows the stump of the left hepatic vein, the middle hepatic vein, and an orifice of the right hepatic duct. This ductal margin was confirmed as histologically negative. The relationship between the right hepatic duct and right portal vein was the same as anticipated preoperatively.

\section{Figure legends}




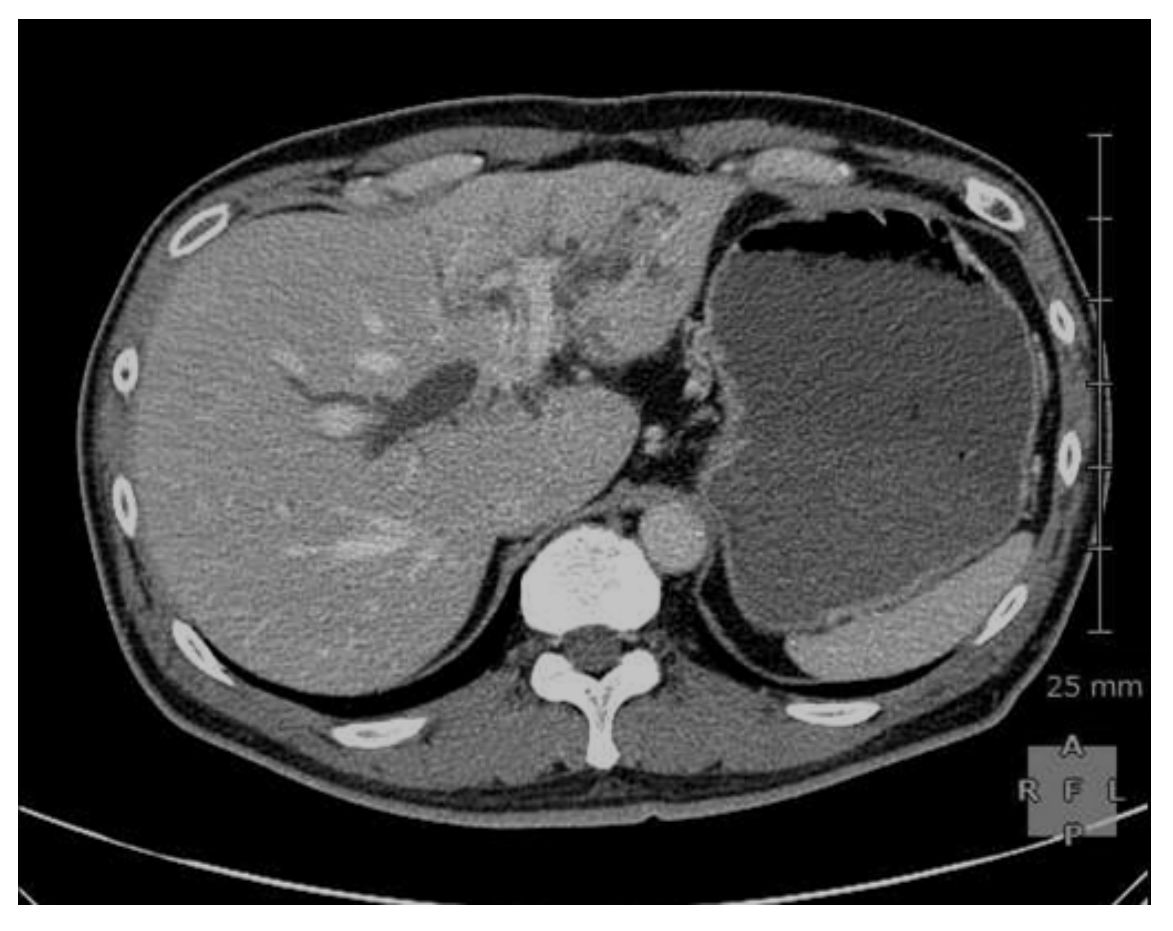

A

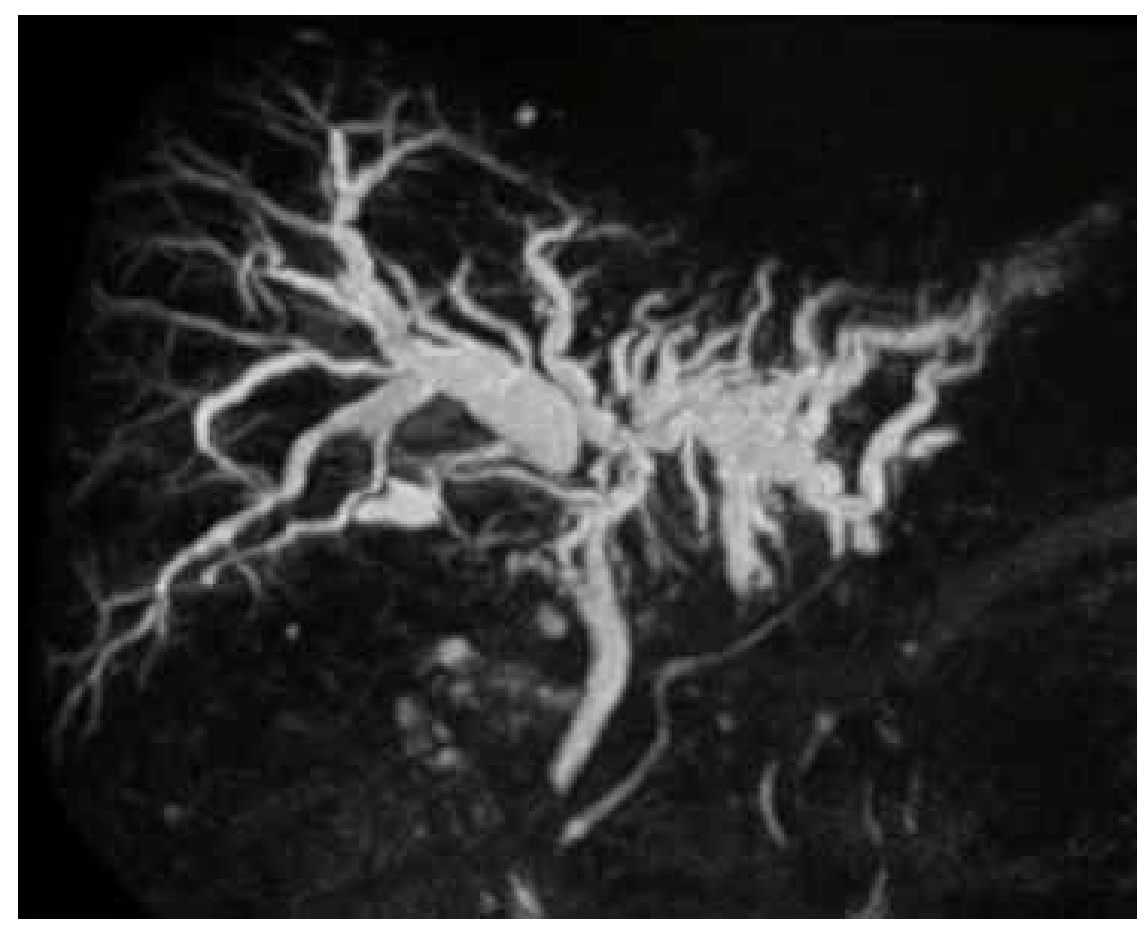

B

Figure 1. 


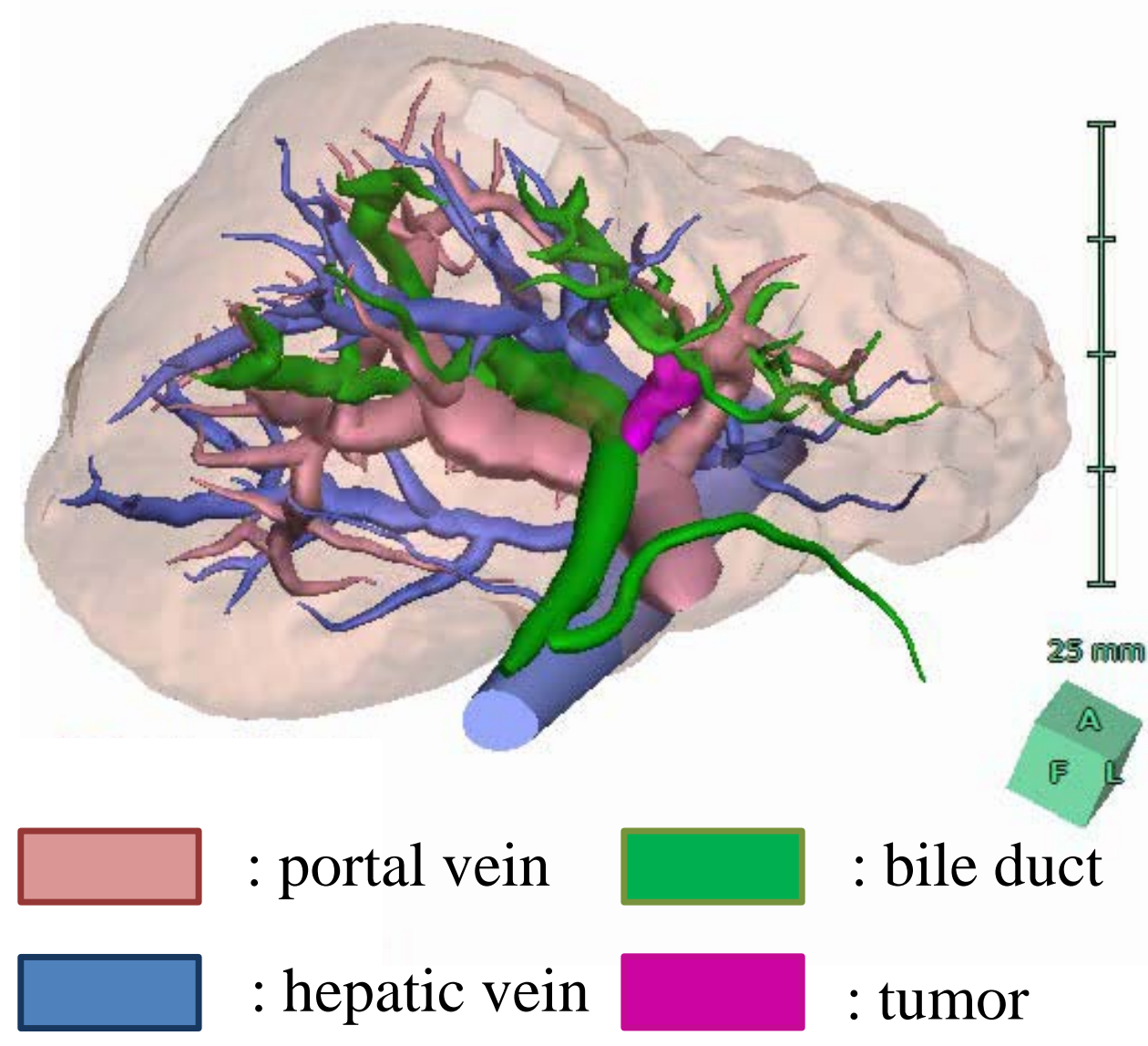

A

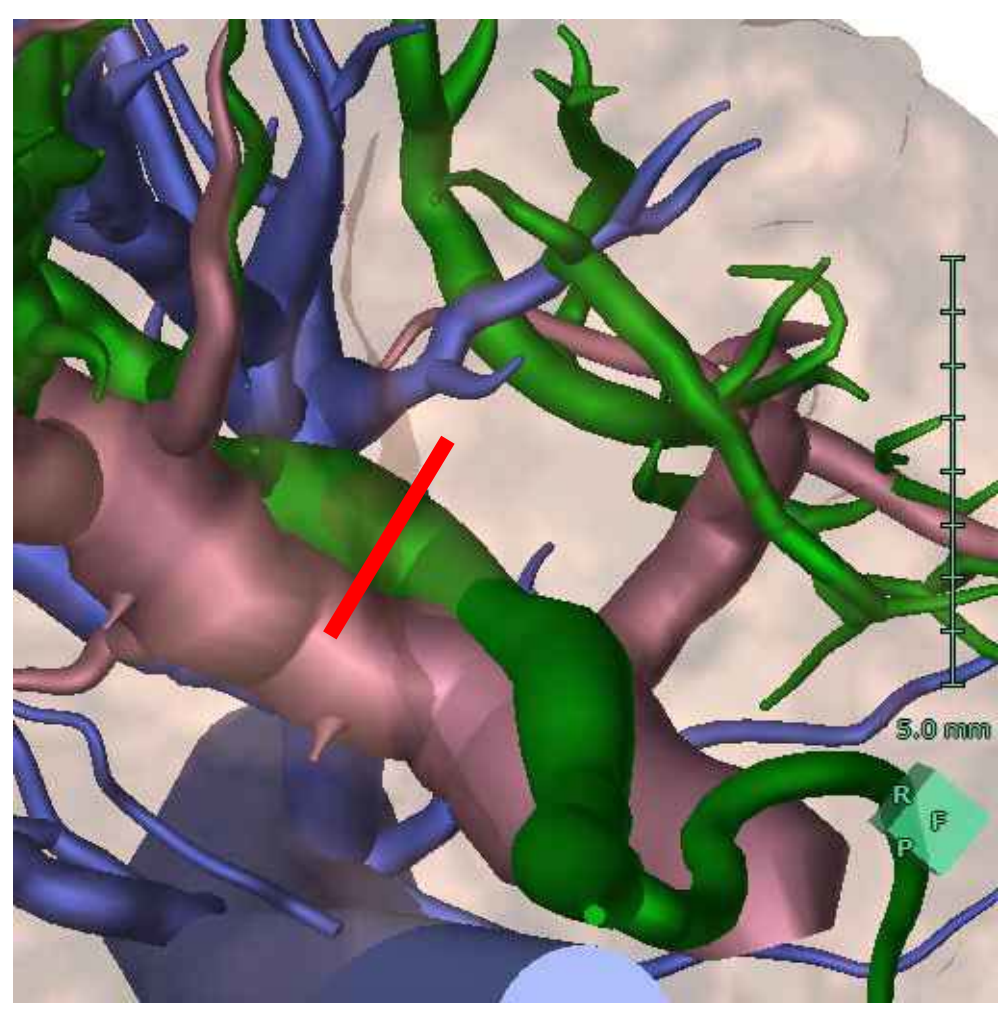

B

Figure 2. 


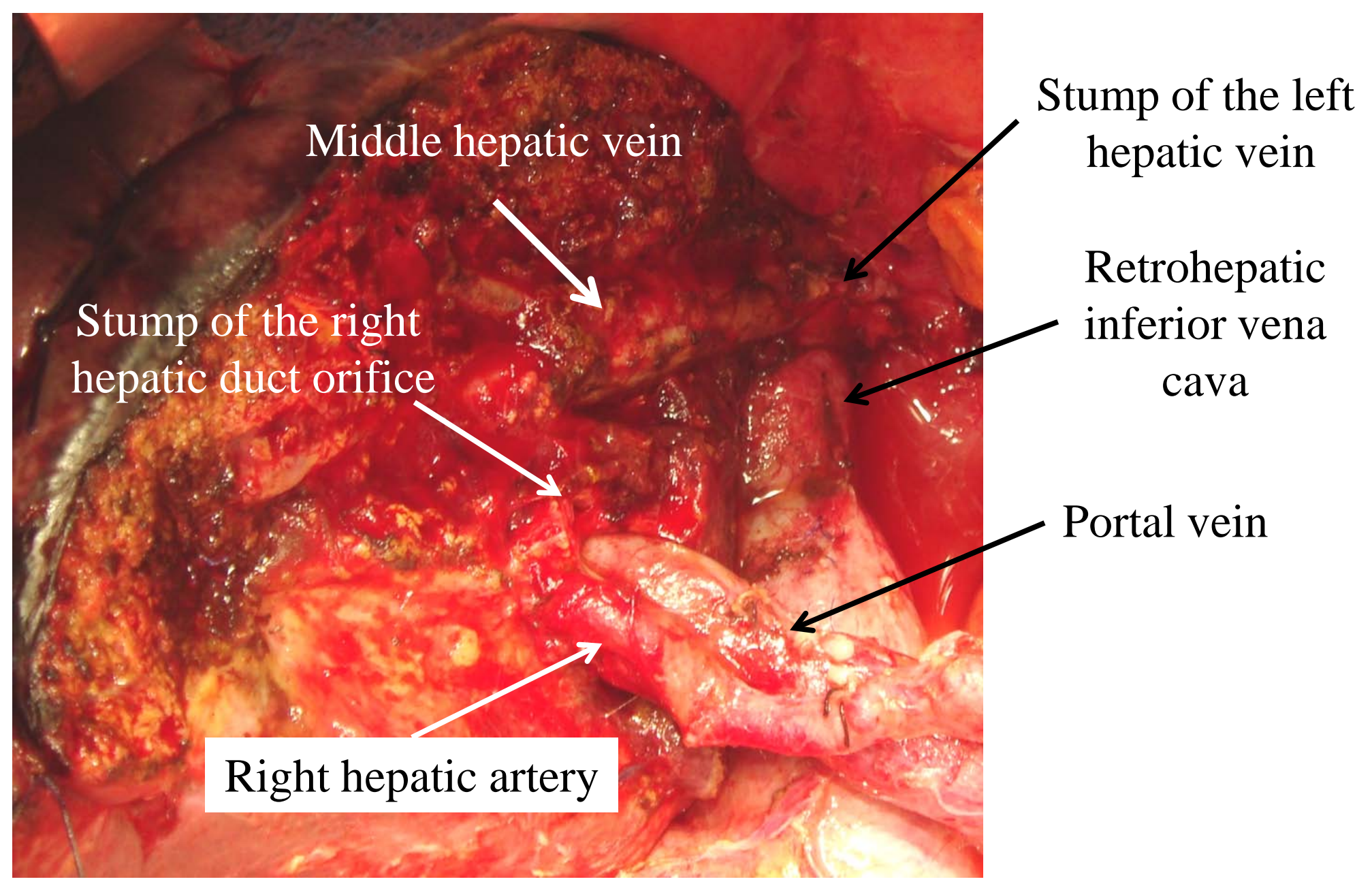

Figure 3. 